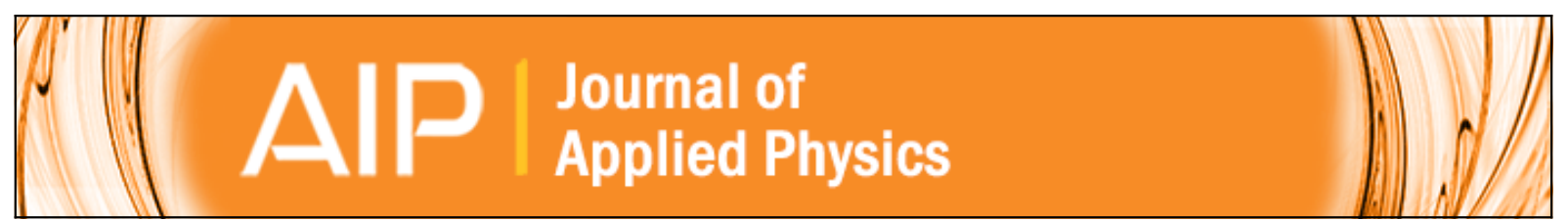

Modulation phenomena in Si nanowire field-effect transistors characterized using noise spectroscopy and gamma radiation technique

S. Pud, J. Li, M. Petrychuk, S. Feste, S. Vitusevich, B. Danilchenko, A. Offenhäusser, and S. Mantl

Citation: Journal of Applied Physics 113, 124503 (2013); doi: 10.1063/1.4795603

View online: http://dx.doi.org/10.1063/1.4795603

View Table of Contents: http://scitation.aip.org/content/aip/journal/jap/113/12?ver=pdfcov

Published by the AIP Publishing

$\underset{\substack{\text { Publoning } \\ \text { PIP }}}{A}$ Re-register for Table of Content Alerts

Create a profile.

Sign up today! 


\title{
Modulation phenomena in Si nanowire field-effect transistors characterized using noise spectroscopy and gamma radiation technique
}

\author{
S. Pud, ${ }^{1}$ J. Li, ${ }^{1}$ M. Petrychuk, ${ }^{2}$ S. Feste, ${ }^{1}$ S. Vitusevich, ${ }^{1, a)}$ B. Danilchenko, ${ }^{3}$ \\ A. Offenhäusser, ${ }^{1}$ and S. Mant ${ }^{1}$ \\ ${ }^{1}$ Peter Grünberg Institute, Forschungszentrum Jülich, Germany \\ ${ }^{2}$ Taras Shevchenko National University, Kiev, Ukraine \\ ${ }^{3}$ Institute of Physics, NASU, Kiev, Ukraine
}

(Received 5 October 2012; accepted 4 March 2013; published online 22 March 2013)

\begin{abstract}
High-quality silicon nanowire (NW) field-effect transistors (FETs) were designed and fabricated. Features of transport and modulation phenomena of the structures were studied using a number of techniques, including noise spectroscopy. Using the $1 / \mathrm{f}$ noise component level, the values of the volume trap densities in gate dielectric are estimated to be around $1 \times 10^{17} \mathrm{~cm}^{-3} \mathrm{eV}^{-1}$. This result proves high quality of the investigated structures. Analysis of Lorentzian noise components of NW samples is used to characterize single trap and its parameters. A strong modulation of carrier concentration in the conducting channel under influence of even single carrier capture event has been revealed. Possibility of fine tuning of the transport properties of the sample with low-dose gamma irradiation has been shown. The gamma radiation treatment of the NW samples was applied as an effective technique to confirm the strong influence of trap charges on conductivity behavior in the channel of NW FETs. The results demonstrate that the modulation effects at the nanoscale enable effective changing of the channel conductivity by single capture events and thus are promising for future information technologies and ultra-sensitive single-molecular sensor applications. (C) 2013 American Institute of Physics. [http://dx.doi.org/10.1063/1.4795603]
\end{abstract}

\section{INTRODUCTION}

Field-effect transistors (FET) based on silicon nanowires (NW) are an important step in the miniaturization of complementary metal-oxide semiconductor (CMOS) devices to obtain state-of-the art devices for information technology. They are the ultimate building blocks for modern electronic devices and biosensors. ${ }^{1-3}$ Utilized for electronics, Si NW FETs allow high-frequency operation and lower power consumption. As biosensors, NWs provide higher sensitivity and spatial resolution compared with conventional planar FETs due to their higher surface to volume ratio. ${ }^{4}$ By considering the transport in silicon NWs, one may see that the current in the Si NW FET is determined by the much lower quantity of carriers than in conventional CMOS FETs due to the small device size. Obviously, this results in higher fluctuations of the conductivity and transport modulation of the NWs. One of the objectives of the biological and bioelectronic utilization of $\mathrm{Si} \mathrm{NWs}$ as sensors is detection of the interaction of a single molecule with the Si NW surface. ${ }^{5}$ To be able to distinguish such a response under the level of native fluctuations of a Si NW, a comprehensive investigation of transport in Si NWs has to be performed. The analysis of the fluctuations in the Si NW FETs combined with I-V characterization contains all the information about performance and transport phenomena in the device. This information is of extreme importance for device development and further improvement of the fabrication technology.

\footnotetext{
a) Author to whom correspondence should be addressed. Electronic mail: s.vitusevich@fz-juelich.de. Tel.: +49 2461 612345. On leave from Institute of Semiconductor Physics, NASU, Kiev, Ukraine.
}

Noise spectroscopy makes it possible to analyze the performance and structure of the samples under study by investigating the fluctuation phenomena. The method itself is a powerful tool for characterizing the dynamic properties of the investigated structure and, hence, for the extraction of information about the origin of noise in the sample. Noise spectroscopy can be used for the analysis of the device quality, transport properties, and improvement of the technology, which is one of the main directions for the development of advanced NW FETs and miniaturization of the elementary basis of CMOSs.

The excess noise spectrum of silicon transistors usually contains generation-recombination (GR) components related to traps with definite energy and a flicker noise component, known as $1 / \mathrm{f}$ noise, as a result of the trap contribution from contact regions and traps related to gate dielectric or mobility fluctuations. ${ }^{6-8}$ The $1 / \mathrm{f}$ noise of metal-oxide semiconductor field-effect transistors (MOSFETs) is usually analyzed in the frame of the McWhorter model, which describes flicker noise by charge carrier trapping/detrapping from the conducting channel to the traps located inside the gate dielectric layer. ${ }^{9}$

The influence of $\gamma$-irradiation on the FETs allows the parameters of the devices to be changed in a controlled way, ${ }^{10,11}$ which may be used for studies and even to improve their characteristics. ${ }^{12}$ In addition, the method can be used to slowly change the parameters of the sample without exerting a strong influence on the device. Thus characterization of the transport in Si NW transistors can be unambiguously performed using $\gamma$-irradiation of the samples with small doses. This allows the material parameters to be changed precisely because the dose of the absorbed radiation can be controlled by accumulation with time. 


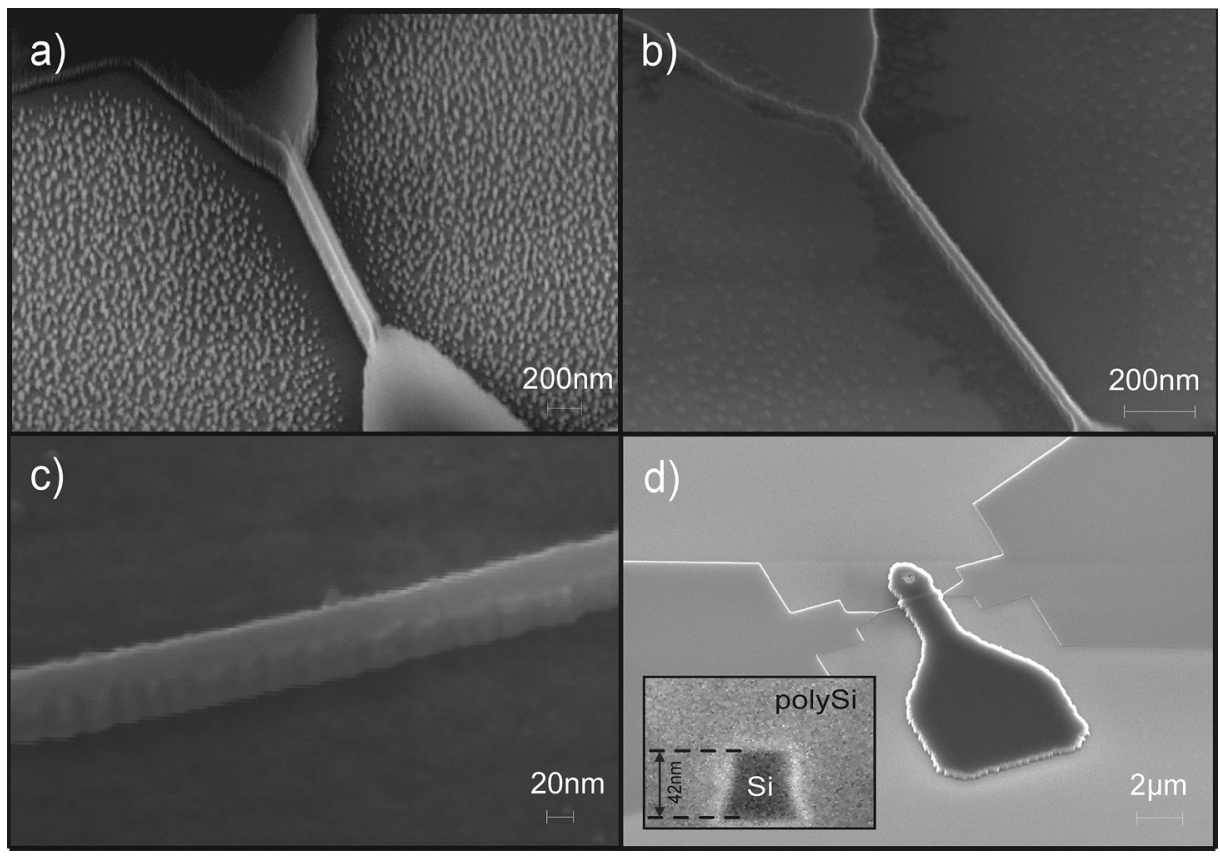

FIG. 1. (a)-(c) SEM image of silicon nanowire device. (d) SEM image of silicon nanowire devices with polysilicon gate. Inset in (d): focused ion beam cut of the wire under study.
Here we present the results of comprehensive transport studies of p-type Si NW FETs. The devices are characterized by noise spectroscopy, which enables us to study the fine transport effects. Using noise spectroscopy, a strong modulation of the channel conductivity is revealed even under the influence of a single trap. Analysis of the 1/f component of the excess noise allowed the value of the volume trap density in the thin dielectric layer to be estimated for measured samples. In these terms, the investigated Si NW FETs demonstrate high quality and improved device performance. The Lorentzian components of the noise spectra are registered and their behavior allows the trapping/detrapping processes on the gate dielectric traps to be characterized. The parameters of the single trap, which generates the random telegraph signal (RTS) noise, in the gate dielectric layer were determined. Investigation of samples after treatment by gamma irradiation with a dose of $10^{4} \mathrm{~Gy}$ confirms the influence of trap charges on conductivity in the channel of NW FETs.

\section{EXPERIMENTAL DETAILS}

The structures under investigation are p-type NW-FETs with a cross-section of $\approx 42 \times 42 \mathrm{~nm}^{2}$ fabricated at Forschungszentrum Jülich using a top-down approach on the basis of $50 \mathrm{~nm}$ SOI wafer $\left(\mathrm{N}_{\mathrm{A}}=1 \times 10^{15} \mathrm{~cm}^{-3}\right.$, buried oxide thickness of $140 \mathrm{~nm}$ ). An N-type polysilicon gate electrode was deposited on the $5 \mathrm{~nm}$ thermally grown $\mathrm{SiO}_{2}$ gate oxide layer, which covered each of the NWs. Source/drain contacts to NWs were formed by ion implantation of boron with an energy of $10 \mathrm{keV}$ and a dose of $1 \times 10^{15} \mathrm{~cm}^{-2}$ followed by rapid thermal annealing. Thus, the samples represent transistors with inversion $p$-channel. The polysilicon covers the NW channel to form a tri-gate (Fig. 1) in the middle part of the NW channel. The tri-gate configuration offers improved gate control over the channel compared to planar geometry. The transistors are in the off-state at zero biases on the front gate and substrate, which plays the role of back gate. The current through the samples can be tuned either by front gate or by back gate voltages.

We investigated noise spectra of NW-FET devices with lengths of $1.5 \mu \mathrm{m}$ and $3 \mu \mathrm{m}$ under different regimes defined by drain-source biases, $V_{D S}$, front-gate voltages, $V_{F G}$, and back-gate voltages, $V_{B G}$. The schematic of the noise measurement setup is shown in Fig. 2. The bias voltages (drainsource and gates) are applied using a battery to avoid circuit fluctuations. Variable resistors allow the values of the applied voltages to be changed. Spectra were acquired in the frequency range from $1 \mathrm{~Hz}$ to $100 \mathrm{kHz}$. The samples were irradiated using a ${ }^{60} \mathrm{Co}$ source of gamma rays with a dose of $10^{4} \mathrm{~Gy}$ and energy of $1.2 \mathrm{MeV}$.

\section{RESULTS AND DISCUSSION}

\section{A. Electric properties before and after gamma irradiation}

The output curves of the investigated samples are shown in Fig. 3. Their behavior is characteristic for metal-oxide semiconductor devices. It should be noted that all noise spectra were measured at low drain bias (around $100 \mathrm{mV}$ ), which provides a linear mode of operation of the transistors in almost the entire range of gate voltages (Fig. 3).

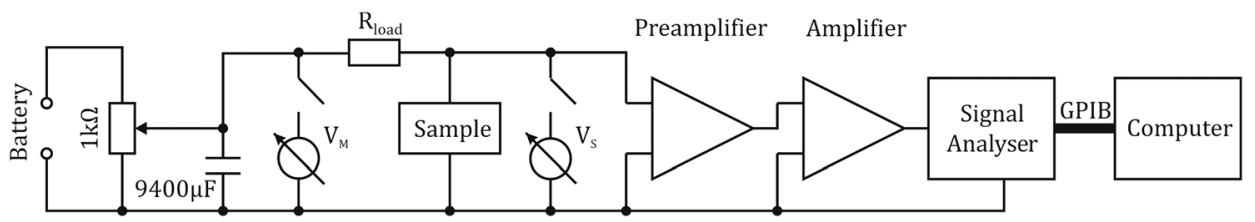

FIG. 2. Schematic of the noise measurement setup. 


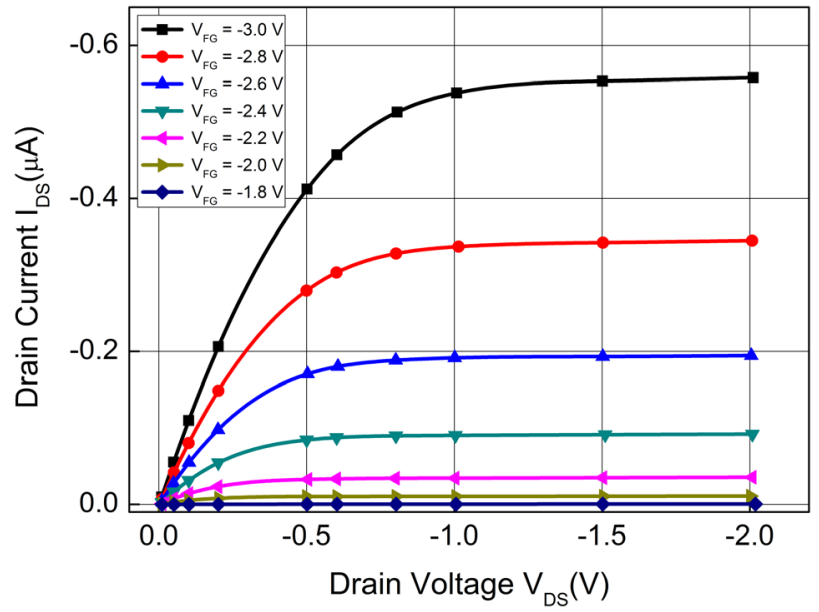

FIG. 3. Typical output characteristics of one of the NW samples under study. Back-gate voltage: $V_{\mathrm{BG}}=0$.

Transfer I-V characteristics for the $1.5 \mu \mathrm{m}$ and $3 \mu \mathrm{m}$ samples are shown in Fig. 4.

The curves reflect the reproducible scaling with decreasing length of the samples. Typical maximum transconductance values $g_{\max }$ for $3 \mu \mathrm{m}$ and $1.5 \mu \mathrm{m}$ samples are $1.3 \times 10^{-7}$ and $2.7 \times 10^{-7} \mathrm{~A} / \mathrm{V}$, respectively. Samples have almost the same threshold voltage $\left(\mathrm{V}_{\mathrm{th}} \sim-1.95 \mathrm{~V}\right)$. The current in the samples shows much weaker dependence on back-gate voltage (Fig. 4(b)) compared with front-gate voltage. This is due to the fact that the buried oxide is significantly thicker than the front-gate oxide layer. It is known that traps in dielectric layers can be affected by using low doses of gamma radiation. $^{21}$ Therefore the characteristics of the samples were investigated both before as well as after gamma radiation treatment. The experimental transfer curves $I_{D S}=f\left(V_{F G}\right)$ of the non-irradiated and irradiated samples at zero substrate bias and different front gate voltages are shown in Fig. 5.

It can be seen that after irradiation treatment the threshold voltage increases and the change in the slope of the transconductance curves is negligibly small. The numerical values of the parameters extracted from Fig. 5 data are summarized in Table I.

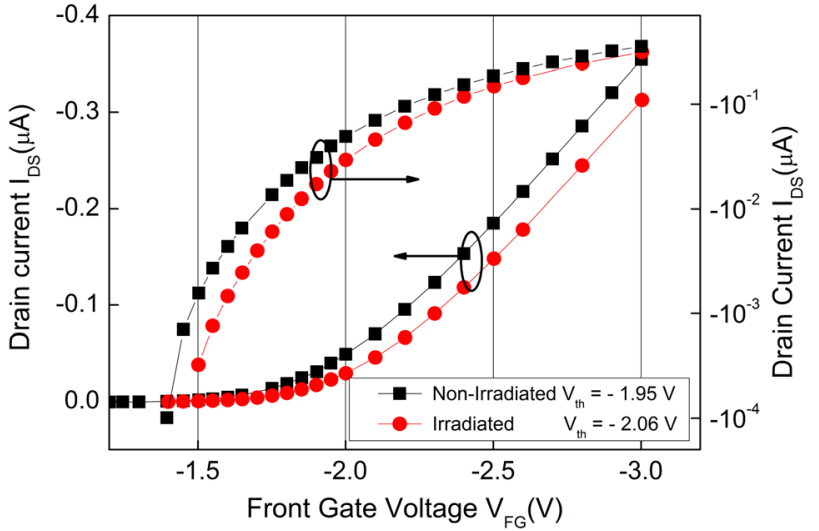

FIG. 5. Typical transfer I-V curves measured for $1.5 \mu \mathrm{m}$ samples at drain bias of $-100 \mathrm{mV}$ and zero back gate voltage before and after irradiation treatment.

As can be seen from Table I and Fig. 5, the irradiation treatment influences the threshold voltage of the samples but does not affect the $g_{\text {max }}$. The data demonstrate that the irradiation changes the surface charge of the $\mathrm{Si} \mathrm{NW}$ without affecting the average scattering times of the impurities inside the channel. The data will be used below.

\section{B. 1/f noise spectroscopy}

Typical drain voltage spectral density of one of the samples measured at several front gate voltages are shown in Fig. 6(a). The spectra contain two noise components: $1 / f$ and Lorentzian shaped.

Using the measurements of noise spectra and I-V characteristics, the volume trap density of the gate dielectric layer can be estimated. ${ }^{6,13}$ This density of traps reflects the quality of the gate dielectric and can be compared for different dielectric layers to optimize the dielectric compositions for miniaturization of the final device. During the process of device downscaling (i.e., from micron width to the submicron scale), at some size even the response of the single traps can be registered. In this case, excess noise contains not only the flicker noise component but also a number of separate Lorentzian components. ${ }^{14,15}$ At a certain level of downscaling the device
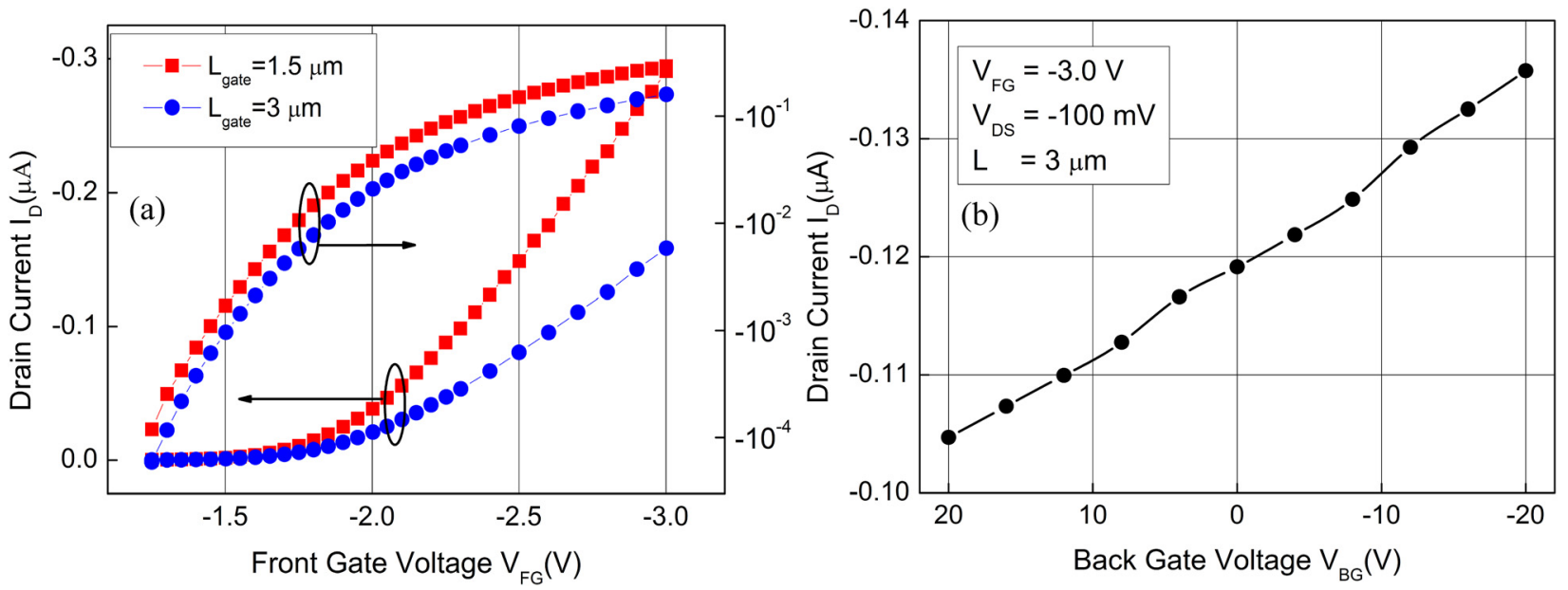

FIG. 4. Transfer curves measured (a) for samples with different lengths $(1.5 \mu \mathrm{m}$ and $3 \mu \mathrm{m})$ at drain bias of $-100 \mathrm{mV}$, zero back gate voltage as a function of front gate voltage; (b) for sample of the $3 \mu \mathrm{m}$ length versus back-gate voltage at drain bias of $-100 \mathrm{mV}$ and front-gate voltage of $-3 \mathrm{~V}$. 
TABLE I. Characteristics of the samples before and after irradiation treatment.

\begin{tabular}{|c|c|c|c|c|}
\hline \multirow{2}{*}{$\frac{\text { Sample gate length }}{\text { Value }}$} & \multicolumn{2}{|c|}{$1.5 \mu \mathrm{m}$} & \multicolumn{2}{|c|}{$3 \mu \mathrm{m}$} \\
\hline & $V_{t h}(\mathrm{~V})$ & $g_{\max }(\mathrm{A} / \mathrm{V})$ & $V_{t h}(\mathrm{~V})$ & $g_{\max }(\mathrm{A} / \mathrm{V})$ \\
\hline Before irradiation & -1.95 & $3.4 \times 10^{-7}$ & -1.95 & $1.8 \times 10^{-7}$ \\
\hline After irradiation & -2.06 & $3.3 \times 10^{-7}$ & -2.06 & $1.8 \times 10^{-7}$ \\
\hline
\end{tabular}

size approaches a limit, at which one or several traps in the dielectric modulate the current. In this case, the signal as a function of time in the device demonstrates the RTS ${ }^{8,14,16-19}$ noise behavior. Analysis of the RTS spectra and time trace allows us to investigate the parameters of the individual traps of nanoscale devices. Individual molecules can play the role of a single trap on the surface of the device, which in this case may be used as the molecule sensor. ${ }^{5,20}$

Equivalent input voltage spectral density is used to calculate volume trap density. $1 / f$ noise current spectral density $\left(S_{I}\right)$ component can be used to calculate the equivalent input voltage spectral density, $S u,{ }^{21,22}$

$$
S_{U}=\frac{S_{I}}{g_{m}^{2}}
$$

where $g_{m}$ is the derivative of the drain current on gate voltage derived from Fig. 4(a). Typical $S_{u}$ dependence on overdrive gate voltage, $V_{G^{-}} V_{t h}$, at frequency of $10 \mathrm{~Hz}$ is shown in Fig. 6(b) for samples of different lengths. The equivalent input power spectral density demonstrates a weak dependence on gate voltage, which indicates that the McWhorter model is applicable for estimating the gate oxide volume trap density. $6,7,23$ The density, $N_{t}$, can be calculated in the frame of the McWhorter model as follows:

$$
N_{t}=\frac{S_{I} \alpha_{t} C_{o x}^{2} W L f}{g_{m}^{2} q^{2} k T},
$$

where $\alpha_{t}$ is the inverse tunneling depth, $C_{o x}$ is the specific gate oxide capacitance, $W$ is the channel width, $L$ is the channel length, $f$ is the frequency, $q$ is the electron charge, $k$ is the Boltzmann constant, and $T$ is the temperature. In our case, $T$ is about $293 \mathrm{~K}$. Here, $\alpha_{t}$ can be estimated using

$$
\alpha_{t}=\sqrt{\frac{2 m_{h}^{*} \varphi_{b}}{\hbar^{2}}},
$$

where $\varphi_{b}$ is the potential barrier between channel and gate dielectric, $m_{h}^{*}$ is an effective hole mass, and $\hbar$ is the reduced Planck's constant. The specific capacity of the gate oxide can be obtained as

$$
C_{o x}=\frac{\varepsilon \varepsilon_{0}}{d_{o x}}
$$

where $\varepsilon$ is the dielectric permittivity of the silicon oxide layer, $\varepsilon_{0}$ is dielectric permittivity of vacuum, $d_{o x}$ is the gate oxide thickness, which in our case is equal to $5 \mathrm{~nm}$.

Using Eq. (2) we estimated the value of the volume trap density for all measured samples. The obtained values are within the range from $1 \times 10^{17} \mathrm{~cm}^{-3} \mathrm{eV}^{-1}$ to $5 \times 10^{17} \mathrm{~cm}^{-3} \mathrm{eV}^{-1}$. It should be noted that these values are about one order of magnitude lower than those obtained for submicron CMOS devices. ${ }^{7}$ By multiplying the obtained values of $N_{t}$ by the oxide thickness $d_{o x}$, the surface trap densities were obtained for our samples. They are expected to be in the range between $5 \times 10^{8} \mathrm{~cm}^{-2} \mathrm{eV}^{-1}$ and $2.5 \times 10^{9} \mathrm{~cm}^{-2} \mathrm{eV}^{-1}$. The densities are much lower than estimated for the thin film dielectric layers of MOS transistors. ${ }^{24}$

\section{Lorentzian components and single traps}

Investigation of the time traces of the noise signal measured on the samples under study showed that a random telegraph signal (RTS) noise is the origin of the low-frequency Lorentzian components in noise spectra. The RTS noise component is a characteristic feature of low-scale samples taking into account that $1 / \mathrm{f}$ noise from the point of view of the McWhorter theory can be represented by superposition of Lorentzian components in the case of a large-sized sample. In the case of large-area samples, the multiple dielectric traps, equally randomly distributed by energy and depths,
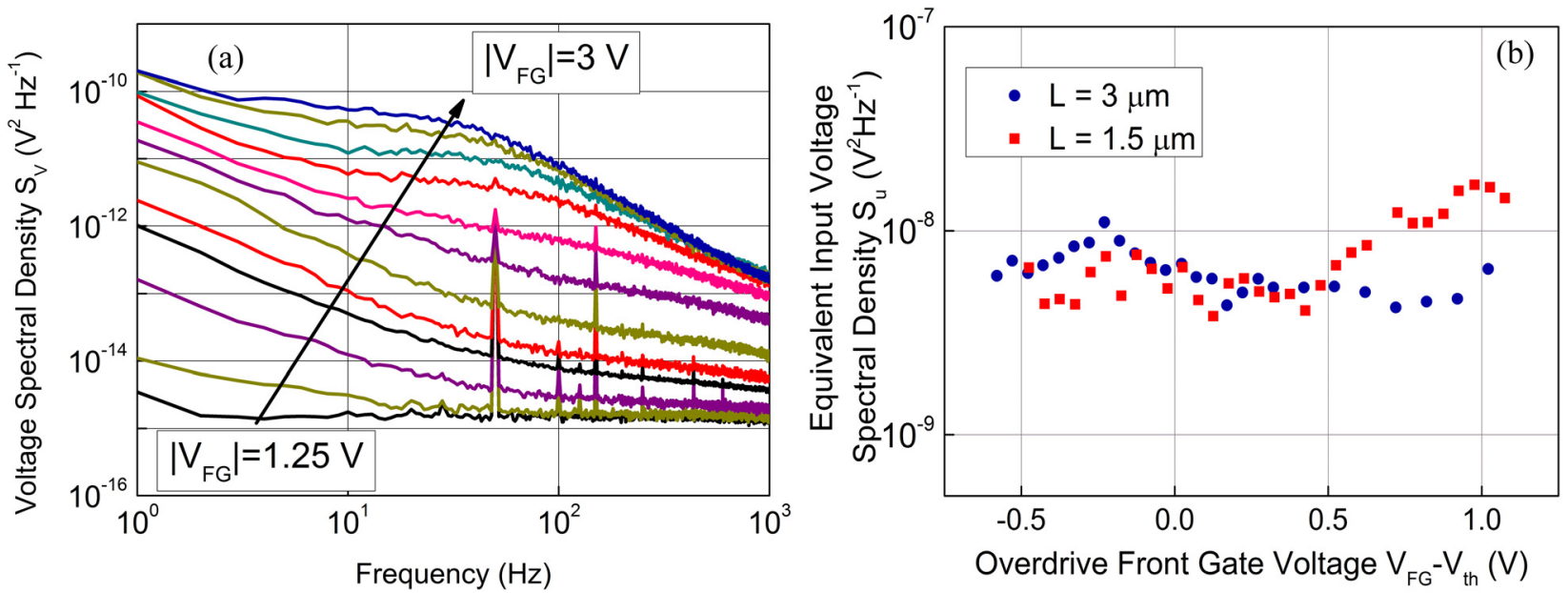

FIG. 6. (a) Noise spectra measured for $3 \mu \mathrm{m}$ sample at different front gate voltages at drain-source bias of $100 \mathrm{mV}$. (b) Equivalent input voltage spectral density for two samples with different lengths estimated for the difference of overdrive gates, $V_{G^{-}} V_{t h}$, at frequency of $10 \mathrm{~Hz}$. 

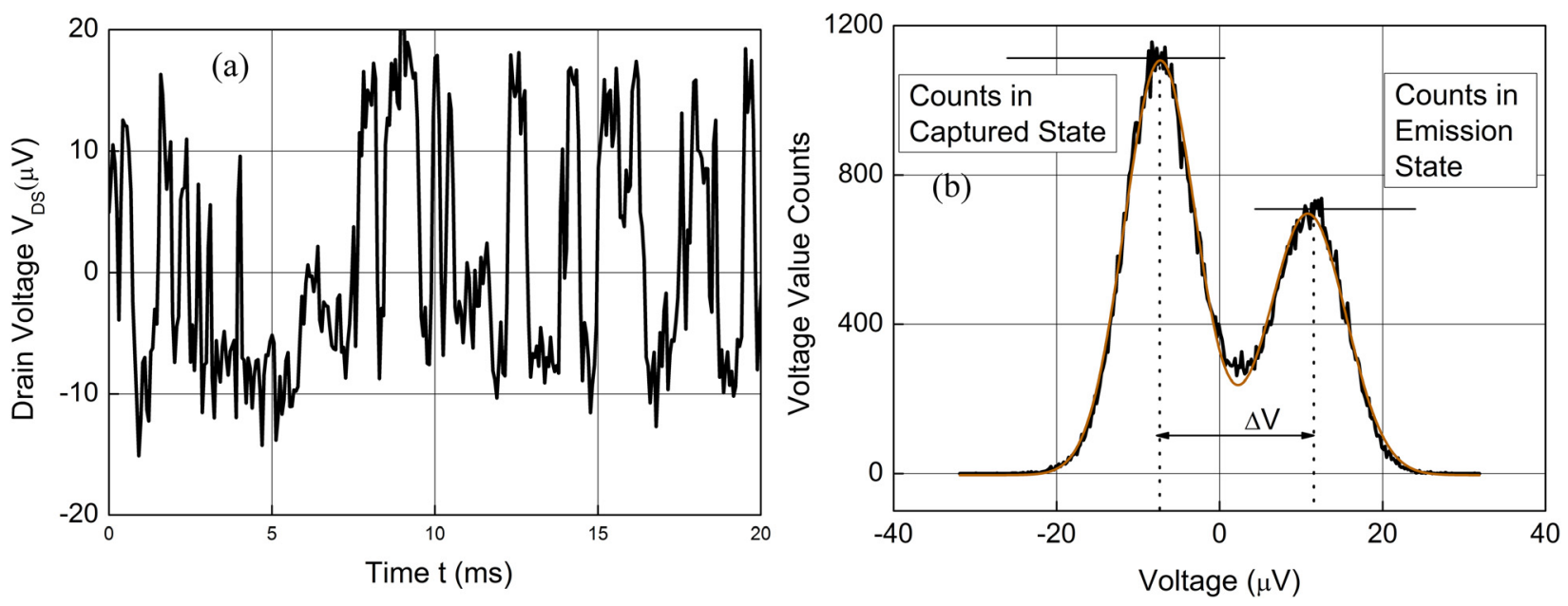

FIG. 7. (a) Random telegraph signal noise time trace measured for $3 \mu \mathrm{m}$ sample at $V_{D S}=-100 \mathrm{mV}, V_{F G}=-3 \mathrm{~V}$. (b) Histogram for the voltage values for the time trace obtained from Fig. 7(a).

result in a $1 / \mathrm{f}$ spectrum of the excess noise. At some characteristic size (tenths of nanometers) of the transistor channel, the noise of the channel can be determined mainly by the single trap in the gate dielectric with energy close to the Fermi level. ${ }^{14,15}$ In this case, the noise may even be observed from the individual oxide trap in the form of RTS that dominates the flicker noise. It should be noted that the above-mentioned individual oxide trap is of the same nature as those traps that are responsible for $1 / \mathrm{f}$ noise in the frame of the McWhorter model. ${ }^{14}$ The spectra of both components, Lorentzian and $1 / \mathrm{f}$ noise, can be analyzed separately. ${ }^{14,25}$

The flicker noise separated into single Lorentzian noise components represents a remarkable opportunity to investigate the single trap properties and predict the properties of samples with a large number of traps in the gate dielectric. Analysis of the RTS time trace allows the capture and emission time constants to be estimated as well as the RTS amplitude for a single trap. Using these data, the capture cross-section of a trap and its position in the gate oxide layer can be calculated. The data obtained can be used to optimize nanoscale Si NW FETs. The RTS noise was registered for the samples with different lengths for a wide range of applied gate voltages. Fig. 7(a) shows the typical time trace of measured RTS noise. We used a statistical method to calculate the capture and emission time on the basis of these data. $^{26}$ If the voltage time trace contains two well-resolved levels, we can construct a histogram of voltage values (shown in Fig. 7(b)).

The histogram of the RTS trace separates into two clearly resolved Gaussian peaks. The ratio of peak heights corresponds to the relation between capture and emission times $\left(\tau_{c}\right.$ and $\left.\tau_{e}\right)$ because the height of each peak is related to the time that the system spends in each state. The distance between the peaks equals the RTS amplitude $\Delta V$, which can be recalculated to the $\Delta I$-difference in current between captured and emission states. The time constant $\tau$ of the Lorentzian spectra that corresponds to the RTS noise can be expressed as ${ }^{14}$

$$
\tau=\frac{\tau_{c} \tau_{e}}{\tau_{c}+\tau_{e}}
$$

Using the $\tau_{c} \tau_{e}$ relation obtained from the histogram and the value of $\tau$ obtained from the spectra, the values of $\tau_{c}$ and $\tau_{e}$ can be obtained.

Histograms of RTS time traces obtained for different front-gate and back-gate voltages are shown in Figs. 8(a) and 8(b). Data of Fig. 8(a) (related to the Lorentzian component of the spectra shown in Fig. 6(a)) demonstrate that the values of capture and emission times depend on the front gate voltage. In contrast, RTS traces of Fig. 8(b) express negligible dependence on the back gate voltage. The fact demonstrates that the trap which results for the RTS modulation of the sample conductivity is located in the top gate dielectric.

The calculated values of $\tau_{c}$ and $\tau_{e}$ are plotted versus the overdrive gate voltage in Fig. 9(a). Obviously, dependencies $\tau_{c}\left(V_{G}-V_{t h}\right)$ and $\tau_{e}\left(V_{G}-V_{t h}\right)$ contain much more information about generation-recombination processes than the resulting time constant of a corresponding Lorentzian spectra component.

\section{Characteristic time constants and position of single traps}

RTS spectroscopy provides advantages for the characterization of trap parameters such as depth, position along the channel, and capture cross-section. ${ }^{14}$ In order to find the position of the trap in the gate oxide we investigated the dependence of the $\tau_{c} \tau_{e}$ relation on front-gate voltage. The depth of the trap in the gate oxide is calculated as follows: ${ }^{16,27}$

$$
x_{t}=-d_{o x} \frac{k T}{q} \frac{d\left(\ln \left(\frac{\tau_{c}}{\tau_{e}}\right)\right)}{d V_{F G}} .
$$

As discussed above, the relation between capture and emission times can be obtained directly from the histograms of the RTS time trace. This relation is shown in Fig. 9(b), obtained for different front gate voltages. The data demonstrate that the logarithm of the $\tau_{c /} \tau_{e}$ depends linearly on the front gate voltage and, hence, Eq. (6) can be used to find the trap depth. Using the $d_{o x}$ as $5 \mathrm{~nm}$ for the samples under study and the linear minimum least squares fit of the data shown in Fig. 9(b), we estimate the trap depth $x_{t}$ to be in the range from $1.65 \mathrm{~nm}$ to $1.85 \mathrm{~nm}$ for all measured samples. 

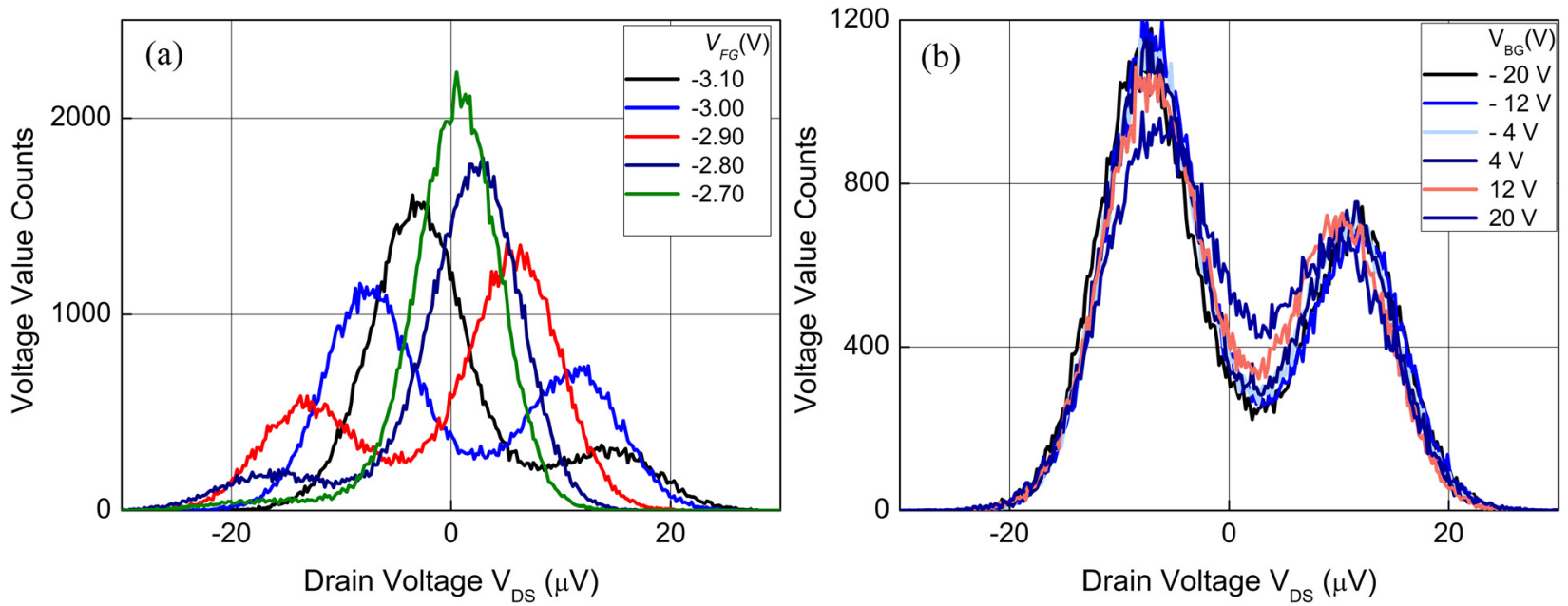

FIG. 8. Histogram of the RTS noise time trace for $3 \mu \mathrm{m}$ sample obtained (a) at zero back-gate voltage and at different front-gate voltages (b) at different backgate voltages and $V_{F G}=-3 \mathrm{~V}$.

The dependence of the time constants of the RTS noise on the source-drain bias contains information about the position of the active trap along the channel length. Therefore, to study the behavior of the active dielectric traps, we investigated the noise of the samples in the nonlinear regime at a fixed front-gate voltage of $V_{F G}=-3.0 \mathrm{~V}$ and different channel biases $V_{D S}=-0.01$ to $-2.0 \mathrm{~V}$ (Fig. 10). In addition, measurements were also made for the reverse polarity of the drain-source bias. Increasing the drain voltage $V_{D S}$ shifts the channel to the nonlinear regime followed by saturation (Fig. 3). With increasing bias voltage on the transistor channel, the gate dielectric voltage, which forms an inverse channel, decreases from $\Phi_{\mathrm{s}}=V_{F G}$ to $\Phi_{\mathrm{s}}=V_{F G}-V_{D S}$. From Fig. 10, it follows that the time constant determined by the RTS noise component is reduced with increasing drain voltage. As we have already discussed above, increasing the drain voltage decreases the $\Phi_{\mathrm{s}}$ near the drain to the value of $V_{F G}-V_{D S}$, and hence it decreases the concentration of carriers in the inversion channel near the drain electrode. These facts indicate that the Lorentzian component of the noise spectra is mainly determined by the emission time constant $\tau_{e}$. Indeed, a decrease of the concentration of free carriers in the inversion channel causes the increase of the probability of emission of free carriers from the centers located in the dielectric layer and, consequently, leads to a decrease in the emission time constant from $2.5 \times 10^{-4}$ to $5.0 \times 10^{-5} \mathrm{~s}$.

In the case of a reverse bias of the Si NW transistor, we can see from Fig. 10 that the emission time constant does not depend on the drain voltage. This fact demonstrates that there are no changes in the concentration of free carriers in the vicinity of the corresponding capture center. Such behavior of the time constant with changes of the drain voltage is only possible if the center is located at a site where the influence of the applied drain voltage is negligibly small (where the gate dielectric voltage $\Phi_{\mathrm{s}}$ is constant and does not depend on $\left.V_{D S}\right)$. Thus we conclude that the dielectric trap, which generates the RTS noise in the investigated sample, is located in the gate dielectric layer close to only one of the ohmic contacts of the Si NW transistor.
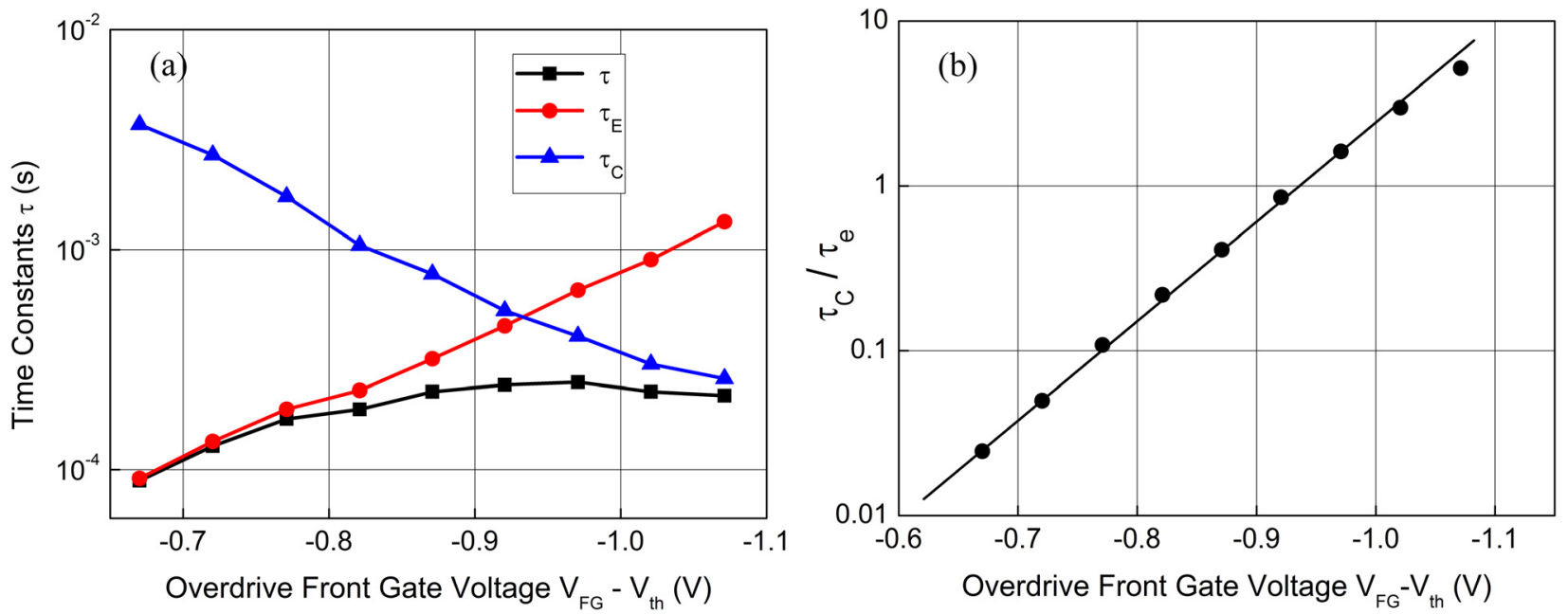

FIG. 9. (a) Time constant of the Lorentzian noise component obtained from Fig. 6(a), calculated emission and capture times as a function of overdrive gate. (b) Calculated relation between capture and emission time plotted versus front-gate voltage. 


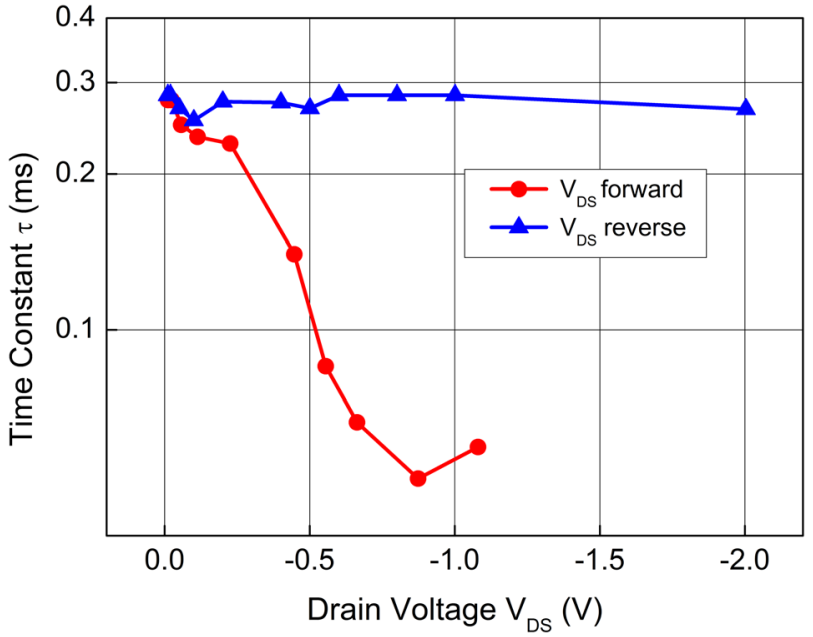

FIG. 10. Time constant of the Lorentzian component of the noise spectra of the sample plotted versus drain voltage at $V_{\mathrm{FG}}=-3.0 \mathrm{~V}, V_{\mathrm{BG}}=0$. Red circles correspond to the GR processes at forward polarity of channel bias; blue triangles correspond to the GR-process at reverse polarity.

\section{E. Modulation effects related to single carrier process}

The current amplitude, $\Delta I$, of RTS fluctuations is an important value, which reflects the impact of a single surface charge on the channel conductivity. ${ }^{28,29}$ It can be obtained from the histograms of voltage fluctuations (see Figs. 7(b) and $8(\mathrm{a})$ ) through dividing of measured RTS voltage, $\Delta V$, by the resistance of the sample in linear regime at certain overdrive voltage. Dependence of the amplitude $\Delta I$ of the RTS fluctuations is shown in Fig. 11. The amplitude is almost constant in the shown range of front-gate voltages and equals $0.20 \pm 0.01 \mathrm{nA}$.

This can be explained as follows. Capture of a free carrier on the fixed trap in the dielectric excludes it from the conductivity. The charged state of the trap results in shielding of part of the channel. At constant voltage $V_{D S}$ applied to the channel, the exclusion of fixed charge in the regime of strong inversion leads to a decrease in the current $\Delta I$. At the same time, the current amplitude does not change. This fact demonstrates that also the mobility remains constant in this

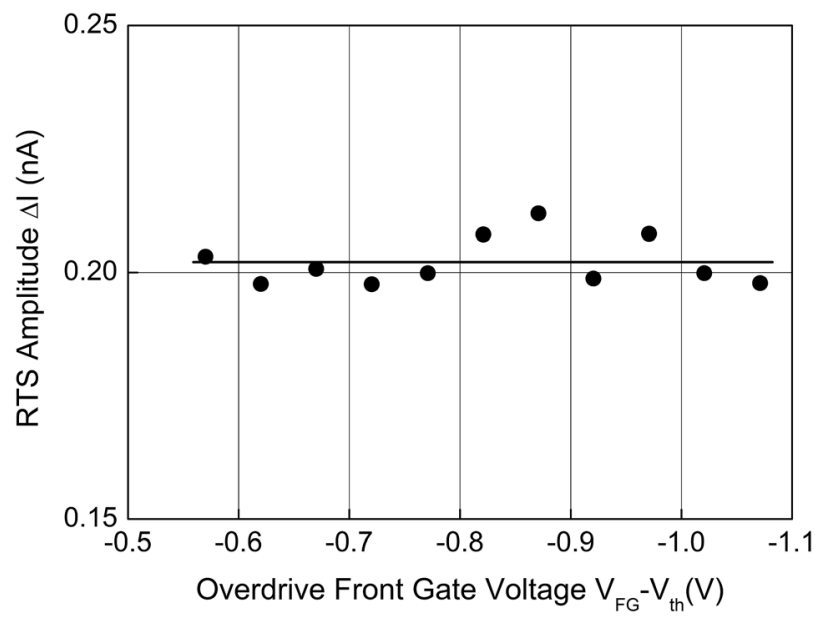

FIG. 11. The RTS noise amplitude dependence on the overdrive front-gate voltage. $V_{\mathrm{BG}}=0, V_{\mathrm{DS}}=-100 \mathrm{mV}$. range of gate voltages. If we assume that only one carrier is excluded from the channel during the capture and that shielding effects are negligible, then we can write

$$
\Delta I=I(N+1)-I(N)=\frac{1}{V} e \mu E S=\frac{1}{V} e \mu \frac{V_{D S}}{L} S=\frac{e \mu V_{D S}}{L^{2}},
$$

where $N$ is the quantity of carriers, $L$ is the length of the channel, $S$ is the area of the channel cross-section, $V$ is the volume of the device, which equals $L S, \mu$ is the mobility of the holes, and $V_{D S}$ is the drain-source bias. Using Eq. (7), the mobility of holes for the sample can be estimated. The calculated value exceeds $1000 \mathrm{~cm}^{2} /(\mathrm{V} \mathrm{s})$, which is obviously too high for the hole mobility in silicon devices. Thus we can conclude that capture of the free carrier on the traps causes a modulating effect on current in the NW channel. Capture of one hole considerably modulates current in the channel (which is equivalent to the exclusion of more than one hole from the transport in the transistor channel). The capture event does not only exclude one carrier from transport but also influences the conductivity of the channel. The conductivity depends on mobility as well as the number of the carrier in the channel. However in our case the obtained values of the capture cross-section (see below) of the single trap appear to be very small $\left(10^{-21} \mathrm{~cm}^{-2}\right)$, and thus mobility modulation due to the capture of a carrier on such a trap may be neglected. At the same time the number of carriers may change as a result of local screening of the charged trap by the carriers of the channel and by plain capacitive effects. $^{30-32}$ This fact demonstrates the possibility of single molecule detection with increased sensitivity using the modulation effect ${ }^{33,34}$ of the channel conductivity in Si NW FET.

\section{F. Single trap properties and parameters}

The obtained RTS parameters allow us to estimate a capture cross-section of the dielectric trap. According to the Shockley-Reed-Hall theory, capture and emission times can be calculated as follows: ${ }^{14}$

$$
\tau_{c}=\frac{1}{\sigma n v_{t h}} \quad \text { and } \quad \tau_{e}=\frac{1}{\sigma n_{1} v_{t h}}
$$

where $\sigma$ is the capture cross-section of the trap, $n$ is the concentration of the carriers in the channel, $n_{1}$ is the the concentration of the carriers in the channel when the Fermi level coincides with the energy of the trap, and $v_{\mathrm{th}}$ is the thermal speed of the carriers. The thermal speed is equal to

$$
\bar{v}=\sqrt{\frac{3 k T}{m^{*}}}
$$

where $m^{*}$ is the effective mass of the carriers. Taking into account that $\mathrm{T}=293 \mathrm{~K}$, the effective mass of the hole is equal to 0.56 of the electron mass $=5.10 \times 10^{-31} \mathrm{~kg}$, the thermal speed is obtained as $1.55 \times 10^{5} \mathrm{~m} / \mathrm{s}$. Then the value of the capture cross-section can be estimated using Eq. (8). It should be noted that $n$ is considered to be a constant value, and the capture cross-section includes tunneling and thermo-activation 

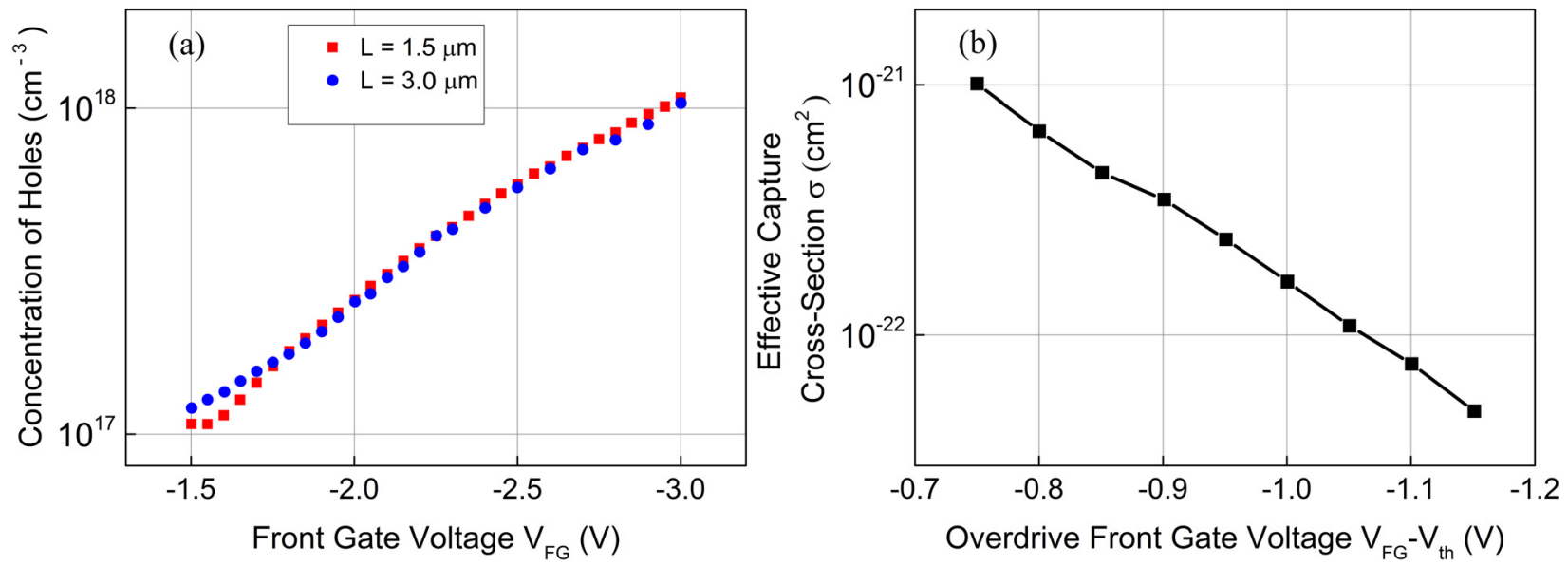

FIG. 12. (a) Calculated concentration of holes as a function of applied gate voltages. (b) Dependence of the calculated effective capture cross-section of the trap on the overdrive gate voltage.

processes, as will be discussed below. Thus the obtained value will be a rough approximation. However, under conditions of high currents this approximation can be accepted. ${ }^{14}$ The concentration and the carrier mobility were estimated using the transfer curve $\mathrm{e}^{21}$ of the $\mathrm{NW}$ transistor with the following relations:

$$
\begin{gathered}
\left.g_{m} \equiv \frac{\partial I_{D}}{\partial V_{G}}\right|_{V_{D}=\text { const }}=\frac{W}{L} \mu C_{o x} V_{D S}, \\
I=n e v S=n e \mu E S \approx n e \mu \frac{V_{D S}}{L} S,
\end{gathered}
$$

where $\mu$ is the carrier mobility, $S=W H$ is the cross-section of the channel, $H$ is the height of the channel, and $E$ is the electric field, which can be estimated in the linear regime using the drain-source bias voltage and the length of the sample.

The characteristics can be obtained

$$
\begin{gathered}
\mu=\frac{g_{m} L}{W C_{t} V_{D}}, \\
n=\frac{I_{D S} C_{o x}}{q g_{m} H} .
\end{gathered}
$$

Using Eq. (12) and data from Fig. 3, we estimated hole mobility for NW samples to be in the range from 120 to 150 $\mathrm{cm}^{2} \mathrm{~V}^{-1} \mathrm{~s}^{-1}$. The calculated values of hole concentrations are shown in Fig. 12(a). It should be noted that the concentrations are the same for the samples with different lengths manufactured on the same wafer.

The fact that the trap is located in the gate dielectric means that the carriers have to overcome the barrier to be captured on the gate oxide trap. Therefore a new multiplier $e^{\frac{E_{b}}{k T}}$ should be added to Eq. (8), which is sequence of the Shockley-Reed-Hall theory ${ }^{14,32}$

$$
\tau_{C}=\frac{e^{\frac{E_{b}}{k T}}}{\sigma n v_{t h}},
$$

where $E_{b}$ represents the energy needed to overcome the barrier from the channel of the device to the trap through the oxide layer. At low concentrations of the carriers in the channel, this energy depends on the gate voltage, but at high drain currents, where the product of capture time $\tau_{c}$ and the drain current $I_{D}$ becomes independent of drain voltage, the value of the trap capture cross-section can be estimated using the Shockley-Reed-Hall theory, particularly without the exponential term. ${ }^{17,29}$ For some of the investigated samples, the condition discussed above can be fulfilled at high front-gate voltages. Thus using Eq. (8), values of $\tau_{c}$, and calculated concentrations, we estimate the capture cross-section of the single trap located in the gate dielectric to be not higher than $2 \times 10^{-21} \mathrm{~cm}^{-2}$, which corresponds to the repulsive trap. ${ }^{14,18}$ Strong dependence of the capture cross-section on the frontgate voltage (see Fig. 12(b)) can be explained by a shift of the centroid of the inversion layer to the $\mathrm{Si} / \mathrm{SiO}_{2}$ interface with increasing surface potential, $\Phi_{\mathrm{s}}{ }^{14,18,30,32}$ Therefore, dependence of the capture cross-section $\sigma$ on gate voltage (Fig. 7(b)) reflects the change of $E_{b}$ as a function of $V_{F G}$. This fact additionally proves that the trap is located in the gate dielectric.

\section{G. Tuning of carrier exchange by $\gamma$-irradiation}

Parameters of the traps can be tuned by $\gamma$-irradiation. ${ }^{35}$ Therefore, utilizing $\gamma$-irradiation treatment allowed us to obtain knowledge about the nature of traps as well as about the mechanisms of influence of such a treatment on the performance of Si NW devices. A shift of threshold voltage (Fig. 5) behavior is demonstrated and discussed above. Changing of the dielectric trap parameters is monitored utilizing noise spectroscopy (Fig. 13).

Comparing the noise values at different frequencies, it may be concluded that the characteristic frequencies of the Lorentzian components shift to the lower frequency after irradiation. The noise of the above-mentioned traps shows similar behavior to the RTS noise. The shift reflects the fact that the capture probability (inversely proportional to time constant $\tau$ ) of traps in the dielectric layer becomes lower near the threshold as well as in strong inversion regimes. As follows from Eq. (14), the value of capture cross section of the trap has decreased if we assume that the values of $E_{b}$ and $n$ are constant. Moreover, the time constant changes by about 

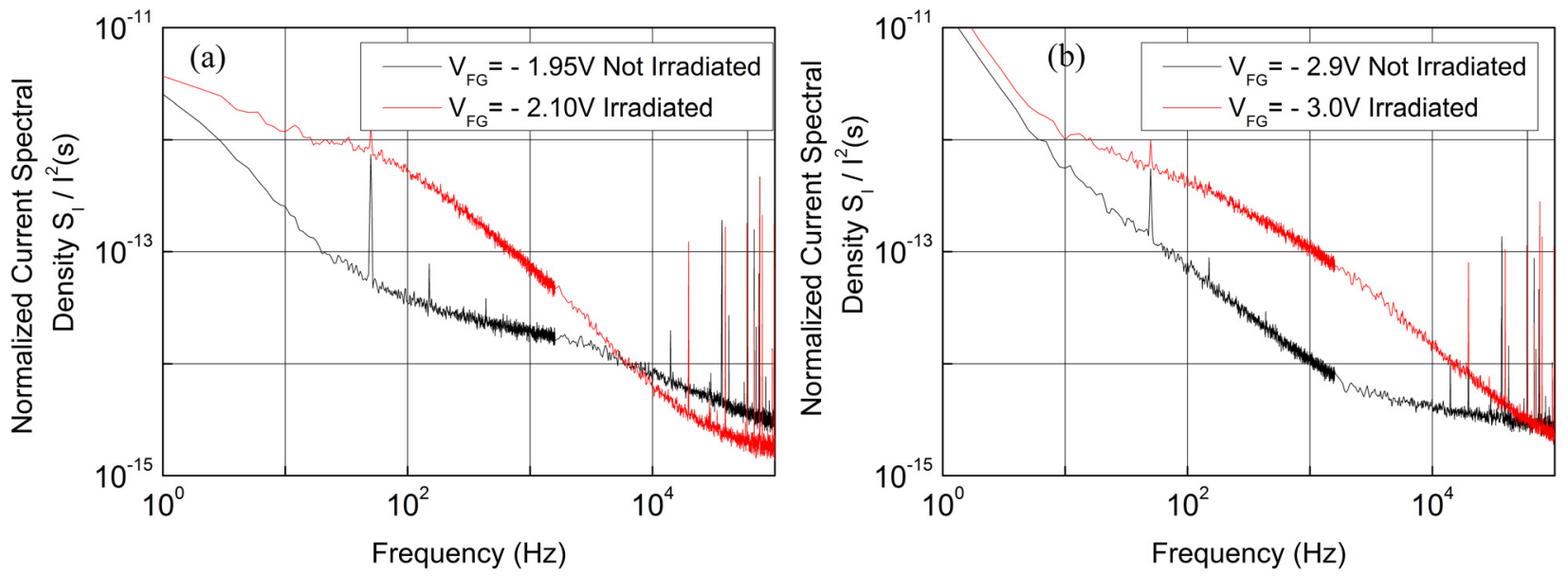

FIG. 13. Normalized current noise spectral density of the Si NW FET measured before and after gamma irradiation near the threshold $\left(V_{\mathrm{FG}}-V_{\mathrm{th}} \approx 0\right)$ and in the regime of strong inversion $\left(V_{\mathrm{FG}}-V_{\mathrm{th}} \approx-1 \mathrm{~V}\right)$, reflecting the characteristic shift of the Lorentzian components.

two orders of magnitude, and the capture cross section $\sigma$ also becomes lower by two orders of magnitude. Such behavior can be explained by the changing of a trap from neutral to repulsive (positively charged). ${ }^{36}$ This fact is additionally confirmed by the shift of the threshold voltage to an increased level corresponding to a more positive potential of $\mathrm{Si} / \mathrm{SiO}_{2}$ interface. Therefore irradiation leads to changes in the charge state of the dielectric traps, which shifts the threshold voltage and, on the other hand, impacts the conditions of the carrier exchange between traps and the conducting channel.

\section{CONCLUSIONS}

Transport properties of p-type silicon nanowires FETs (with a cross-section of $\approx 42 \times 42 \mathrm{~nm}^{2}$ fabricated at Forschungszentrum Jülich) utilizing noise spectroscopy are studied. The devices possess low excess noise level. The values of volume trap density obtained from the level of input voltage spectral density are much lower than those obtained for conventional CMOS devices. The devices with different channel lengths have almost the same input voltage spectral density indicating that the influence of contact effects on the performance of the investigated devices can be neglected. The nature of the changes in the defect structure of the $\mathrm{Si}$ NW samples under low doses of gamma radiation was analyzed. In particular, our measurements demonstrate that low doses of gamma irradiation result in a shift the threshold voltage, without any influence on the scattering times inside the channel and the mobility. Low doses of gamma irradiation change the charge state of the traps located in the gate dielectric, which confirms that the origin of noise is related to gate dielectric traps. Analysis of the registered RTS noise component reveals that a single trap is located near one of the ohmic contacts in the gate dielectric. Estimated parameters of the trap and its behavior demonstrate that even a single carrier process in the gate of the NW transistor considerably modulates current in the channel. These results are promising for advanced control of the channel transport in NW FETs, including the possibility of single molecule detection with increased sensitivity using the modulation effect of the channel conductivity in Si NW FET.
${ }^{1}$ W. Lu, P. Xie, and C. M. Lieber, IEEE Trans. Electron Devices 55, 2859 (2008).

${ }^{2}$ H. S. Song and T. H. Park, Biotechnol. J. 6, 1310 (2011).

${ }^{3}$ X. Liu, Y. Long, L. Liao, X. Duan, and Z. Fan, ACS Nano 6, 1888 (2012).

${ }^{4}$ T. Kong, R. Su, B. Zhang, Q. Zhang, and G. Cheng, Biosens. Bioelectron. 34, 267 (2012).

${ }^{5}$ J. Hahm and C. M. Lieber, Nano Lett. 4, 51 (2004).

${ }^{6}$ G. Ghibaudo, F. Balestra, and J. Brini, Phys. Status Solidi 124, 571 (1991).

${ }^{7}$ J. Jomaah, M. Fadlallah, and G. Ghibaudo, Adv. Mater. Res. 324, 441 (2011).

${ }^{8}$ Z. Celik-Butler, IEE Proc.: Circuits Devices Syst. 149, 24 (2002).

${ }^{9}$ S. Christensson, I. Lundström, and C. Svensson, Solid-State Electronics 11, 797 (1968).

${ }^{10}$ B. Loncar, S. Stankovic, K. Stankovic, and B. Jovanovic, Progress in Electromagnetics Research Symposium Proceedings, Xi'an, China, 22-26 March 2010, pp. 1193-1198.

${ }^{11}$ M. James, U. S. Army, and D. Laboratories, vol. NS-27 1739 (1980).

${ }^{12}$ A. M. Kurakin, S. A. Vitusevich, S. V. Danylyuk, H. Hardtdegen, N. Klein, Z. Bougrioua, B. A. Danilchenko, R. V. Konakova, and A. E. Belyaev, J. Appl. Phys. 103, 083707 (2008).

${ }^{13}$ S. Villa, G. De Geronimo, A. Pacelli, A. L. Lacaita, and A. Longoni, Microelectron. Reliab. 38, 1919 (1998).

${ }^{14}$ M. J. Kirton and M. J. Uren, Adv. Phys. 38, 367 (1989).

${ }^{15}$ K. K. Hung, P. K. Ko, C. Hu, and Y. C. Cheng, IEEE Electron Device Lett. 11, 90 (1990).

${ }^{16}$ C. Leyris, F. Martinez, A. Hoffmann, M. Valenza, and J. C. Vildeuil, Microelectron. Reliab. 47, 41 (2007).

${ }^{17}$ N. Lukyanchikova, M. Petrichuk, N. Garbar, E. Simoen, and C. Claeys, Microelectron. Reliab. 38, 1561 (1998).

${ }^{18}$ N. Lukyanchikova, M. Petrichuk, N. Garbar, E. Simoen, and C. Claeys, Microelectron. Eng. 48, 185 (1999).

${ }^{19}$ S. Yang, K. H. Yeo, D.-W. Kim, K. Seo, D. Park, G. Jin, K. Oh, and H. Shin, in 2008 IEEE International Electron Devices Meeting, 15-17 December 2008, 1 (2008).

${ }^{20}$ Y. He, S. Su, T. Xu, Y. Zhong, J. A. Zapien, J. Li, C. Fan, and S.-T. Lee, Nano Today 6, 122 (2011).

${ }^{21}$ S. M. Sze, Physics of Semiconductor Devices, 2nd ed. (WileyInterscience, 1981), pp. 433-456.

${ }^{22}$ L. K. J. Vandamme and D. Rigaud, IEEE Trans. Electron Devices 41, 1936 (1994)

${ }^{23}$ G. Ghibaudo and O. Roux-dit-Buisson, in 24th European Solid State Device Research Conference (ESSDERC'94), 11-15 September 1994, pp. 693-700.

${ }^{24}$ G.-W. Lee, J.-H. Lee, H.-W. Lee, M.-K. Park, D.-G. Kang, and H.-K. Youn, Appl. Phys. Lett. 81, 2050 (2002).

${ }^{25}$ F. Daugé, J. Pretet, S. Cristoloveanu, A. Vandooren, L. Mathew, J. Jomaah, and B.-Y. Nguyen, Solid-State Electron. 48, 535 (2004).

${ }^{26}$ Y. Yuzhelevski, M. Yuzhelevski, and G. Jung, Rev. Sci. Instrum. 71, 1681 (2000).

${ }^{27}$ H. Lee, Y. Yoon, S. Cho, and H. Shin, IEICE Trans. Electron. E90-C, 968 (2007).

${ }^{28}$ E. Simoen, B. Dierickx, B. De Canne, F. Thoma, and C. Claeys, Appl. Phys. A 58, 353 (1994). 
${ }^{29}$ E. Simoen, C. Claeys, N. Lukyanchikova, M. Petrichuk, and N. Garbar, Solid State Phenom. 69-70, 467 (1999).

${ }^{30}$ M. Schulz, J. Appl. Phys. 74, 2649 (1993).

${ }^{31}$ O. Roux-dit-Buisson, G. Ghibaudo, and J. Brini, Solid-State Electron. 35, $1273(1992)$

${ }^{32}$ H. H. Mueller, D. Woürle, and M. Schulz, J. Appl. Phys. 75, 2970 (1994).
${ }^{33}$ G. Zheng, X. P. A. Gao, and C. M. Lieber, Nano Lett. 10, 3179 (2010).

${ }^{34}$ C. Li, E. Krali, K. Fobelets, B. Cheng, and Q. Wang, Appl. Phys. Lett. 101, 222101 (2012)

${ }^{35}$ V. S. Lysenko, I. P. Tyagulsky, I. N. Osiyuk, and A. N. Nazarov, Quantum Electron. Optoelectron. 10, 34 (2007), available at http://journal-spqeo.org.ua/ n2_2007/n2_2007_contents.htm.

${ }^{36}$ M. Odalović and D. Petković, Mater. Sci. Forum 555, 147 (2007). 